\title{
Impaired Inhibition of Conditioned Responses Produced by Subchronic Administration of Phencyclidine to Rats
}

\author{
J. David Jentsch, Ph.D. and Jane R. Taylor, Ph.D.
}

Several recent investigations have suggested that an important function of the frontostriatal system is inhibitory response control, and we previously reported that subchronic exposure to phencyclidine $(P C P)$ produced deficits in inhibitory control in monkeys. The current studies were designed to examine whether subchronic administration of $P C P$ to rats would subsequently affect the ability to inhibit conditioned responses when relationships between reward and stimuli of affective significance change. First, the effects of long-term exposure to PCP on acquisition of a novel, concurrent discrimination or reversal learning were assessed; PCP-treated rats were selectively impaired in the ability to acquire the reversal of an already-learned stimulus-reward association. Furthermore, there were no effects of PCP treatment on the learning of a novel instrumental response; however, PCP-treated rats produced more responses during extinction of instrumental responding than did control subjects. Finally, PCP-treated rats produced more responses for a conditioned reinforcer than did control rats. These data suggest that PCP-treated rats are impaired in their ability to modulate behavior based upon new or changing information about stimulus-reward associations, possibly due to an inability to inhibit conditioned responding towards incentive stimuli. These effects may have relevance to mental disorders involving affective impairments and impulsivity, including schizophrenia, obsessive-compulsive disorders, and drug abuse.

[Neuropsychopharmacology 24:66-74, 2001] (c) 2000 American College of Neuropsychopharmacology. Published by Elsevier Science Inc.
KEY WORDS: NMDA; Medial frontal cortex; Response inhibition; Working memory; Conditioned reinforcer; Discrimination learning; Reversal learning; Extinction; Reward

One of the proposed functions of the prefrontal cortex and its projection sites within the striatal complex is inhibitory response control (Milner 1963; Mishkin 1964; Robbins 1996). Studies in non-human primates have suggested that subjects with lesions within the ventral

From the Department of Psychiatry (JDJ, JRT), Yale University School of Medicine, New Haven, CT.

Address correspondence to: Jane R. Taylor, Ph.D., Department of Psychiatry, Yale University School of Medicine, 333 Cedar Street, PO Box 208068, New Haven, Connecticut 06520-8068.

Received 5 June 1999; revised 23 June 2000; accepted 5 July 2000. regions of frontal cortex exhibit impaired ability to withhold responses in Go/No-go or operant extinction tests. Moreover, monkeys with lesions of the frontal cortex exhibit abnormal control of behavior by previously-reinforced stimuli in discrimination reversal tasks and show deficits in object retrieval/detour paradigms where animals have to retrieve a reward from a transparent box by making a reaching detour while inhibiting reaching directly at the reward (Butter 1968; Iversen and Mishkin 1970; Rosenkilde 1975; Diamond 1988; Dias et al. 1996a,b, 1997; Wallis et al. 1997). Likewise, frontal cortical lesions in rats can produce reversal-learning deficits (Divac 1971; Kolb et al. 1974; Bussey et al. 1997).

In each of these tasks, the performance impairments can be described as increased control of behavior by "pre-potent" stimuli (i.e., stimuli with previous motiva- 
tional significance). These pre-potent stimuli can be unconditioned reinforcers or can acquire motivational significance by contingent association with primary reward, thus becoming second-order, conditioned reinforcers. The Go/No-go and extinction deficits may reflect an inability to inhibit the conditioned, pre-potent tendency to respond, per se, while deficits on the object retrieval/detour task may result from "enhanced appetitive effects of reward over behavior" (Dias et al. 1996b, page 884). Moreover, in reversal tasks, the originally reinforced stimulus likely acquires conditioned-reinforcing properties by contingent association with reward.

These deficits in monkeys are analogous to those observed in humans with damage within the ventral frontal cortex. Lesions to the orbitofrontal cortex in humans results in the preference for immediate, inefficient rewards, over delayed but more advantageous rewards, as measured by "option-outcome" procedures (Damasio 1996). Likewise, lesions to more dorsal regions of the prefrontal cortex impair performance of Stroop and Wisconsin Card Sorting tests, both of which require intact "inhibitory control" (Milner 1963).

An elegant method for evaluating the incentive qualities of stimuli and the control of behavior by conditioned stimuli is the "acquisition of a new response" (Hill 1970; Robbins 1975, 1976; Mackintosh 1974; Taylor and Robbins 1984). In this paradigm, rats previously trained to associate a neutral stimulus (e.g., tone + light pairing) with an unconditioned reinforcer (e.g., water) are tested to determine whether they would learn a novel instrumental response when rewarded with the tone + light pair alone. Since the animal no longer receives the primary reward, the acquisition of the novel response is reflective of the motivational significance of the second-order, or conditioned, reinforcer (tone + light). Using another paradigm to assess the motivational valence of conditioned reinforcers paired with cocaine infusion, Everitt and colleagues have recently demonstrated that lesions of the medial frontal cortex of rats augment responding for a second-order reinforcer and impair extinction of a previously reinforced response (Weissenborn et al. 1997). This effect may reflect behavioral disinhibition, or increased control of behavior by the conditioned stimulus.

The effects of long-term exposure to the psychotomimetic NMDA/glutamate receptor antagonist, phencyclidine $(\mathrm{PCP})$, on cortical cognitive function have recently been investigated. Subchronic exposure to PCP was found to impair performance of an object retrieval/ detour task by monkeys (Jentsch et al. 1997a, 2000) and these behavioral deficits were found to correlate with dopaminergic deficits in selected regions of prefrontal cortex (Jentsch et al. 1999). As this test appears to be sensitive to frontal cortical function and inhibitory control, these impairments may reflect increased control of behavior by appetitive rewards. In the current studies, the effects of long-term PCP treatment on several behavioral tests sensitive to inhibitory control were assessed. Rats were treated repeatedly with saline or PCP, and after withdrawal, were evaluated for discrimination acquisition or reversal or the acquisition and extinction of an instrumental response. Finally, responding for a conditioned reinforcer was assessed in control and PCP-treated rats. The dose of PCP, schedule of its administration and period of withdrawal has been shown previously to result in prefrontal cortical dopaminergic and behavioral impairments in rats (see Jentsch et al. 1997b, 1998).

\section{MATERIALS AND METHODS}

All procedures were previously approved by the institutional IACUC. All subjects were maintained under conditions consistent with the USDA, Yale University School of Medicine, NIH and the Society for Neuroscience guidelines for the care and use of laboratory animals.

\section{Animals}

Subjects were male Sprague-Dawley rats (CAMM, Wayne, NJ), weighing 250-275 g before behavioral testing. All animals $(n=52)$ were housed in pairs in a temperature-controlled environment under 12:12-hr lightdark conditions. For discrimination acquisition and reversal learning studies, water was available ad libitum, whereas food availability was restricted in order to maintain the subjects at $85 \%$ of their free-feeding weights. For the operant tests (schedule-controlled behavior and conditioned reinforcer testing), the rats were water-deprived but had ad libitum access to food. These subjects received water during the testing session and had access to water for $30 \mathrm{~min}$ per day, $30 \mathrm{~min}$ after the daily testing session such that they were on a 23-hour restriction schedule.

\section{PCP Treatment}

At varying times during testing, rats were treated repeatedly with PCP (Research Biochemicals Inc., Natick MA; 5 $\mathrm{mg} / \mathrm{kg}$, i.p. twice-daily for seven days) or saline $(0.1 \mathrm{ml} /$ $\mathrm{kg}$ i.p. twice-daily for seven days). Injections were given at approximately 9 a.m. and 9 p.m., daily. A 7-day withdrawal period after the final drug administration was always allowed before testing commenced. The dose of PCP and procedures were based on several previous published studies in rodents (see Jentsch et al. 1997b, 1998).

\section{Visual Discrimination Testing}

Pre-Training. All rats in the reversal experiments were initially exposed to a standard, black T-maze (see 
Murphy et al. 1996 for dimensions). Rewards (miniature chocolate chips) were available non-contingently in both arms. Each subject was allowed to explore the maze until 10 chocolate chips were consumed or 15 minutes had passed. This habituation phase was performed on two consecutive days.

General Testing Methodology. All subjects were trained twice a week during 20-trial sessions by an experimenter who was blind to treatment conditions. Responses (i.e., arm choices) and latencies were manually recorded by the observer. For half the subjects, the black arm was the positively reinforced stimulus $\left(\mathrm{SR}^{+}\right)$, whereas the white arm was the $\mathrm{SR}^{+}$for the other subjects. Placing 1 $\mathrm{mm}$ thick Plexiglas inserts into the alleys altered arm color. The position of the colored inserts in the right versus left arms was pseudo-randomly varied throughout the 20 daily trials, but the colors were always evenly distributed between the left and right arms over the course of the 20 trials. Thus, the color, and not the spatial position, of the arms was associated consistently with reward.

Novel Discrimination Learning (Acquisition). In the first experiment, a group of subjects was repeatedly exposed to PCP or saline following the two-day habituation procedure. One week after the final drug administration, testing on the acquisition of a novel discrimination was initiated; the subjects were placed in the start box and allowed a maximum of $3 \mathrm{~min}$ to enter either colored arm. If the subject chose the $\mathrm{SR}^{+}$(the correct colored arm), a reward was placed in a food-well at the end of the arm. After trial completion, the rat was returned to the start box for approximately $5 \mathrm{sec}$ (sufficient time to wipe the maze choice point and inserts with $70 \%$ alcohol and change, if necessary, the position of the colored inserts) before the next trial commenced. Training was performed twice-weekly for a total of five training sessions (a total of 100 trials).

Reversal Learning. In a second experiment, drug-naive subjects were first habituated to the maze and then trained on the acquisition of a novel discrimination during a five-day training period (as above). The subjects were then repeatedly exposed to PCP or saline. Subsequently, they were given a one-week washout period. All the subjects were then tested on a reversal condition, i.e., the reinforcement contingencies were switched such that the color previously not associated with reward was now the reinforced stimulus (the color previously designated as the $\mathrm{SR}^{+}$became the $\mathrm{SR}^{-}$, and vice versa). The rats were tested daily in six separate $20-$ trial sessions (120 trials total).

\section{Fixed Ratio Performance and Extinction}

In the third experiment, a group of naive rats was repeatedly exposed to $\mathrm{PCP}$ or saline, and following a one- week withdrawal period, the subjects were waterdeprived according to the 23-hour restriction schedule. Two days later, the subjects were placed in an operant chamber equipped for liquid reinforcement (Med Associates, Inc., E. Fairfield, VT) with two operable levers and a liquid dipper within a photocell-equipped magazine (see Taylor and Horger 1999 for details). For the first two 30-min training sessions, a total of 20 reinforcers were randomly delivered to the magazine via a motorized dipper, and this delivery was signaled (presaged by $5 \mathrm{sec}$ ) by a SonAlert tone and the co-activation of a light above the magazine (tone + light compound CS). Head entries during the CS and US intervals ("appropriate entries") and non-CS + US times ("inappropriate entries) were recorded. On the following days, a fixed ratio schedule of reinforcement for lever pressing was instituted, with reward delivered only upon responding on one of the two levers (the other was recorded from but was inactive).

The reinforcement schedule was progressively increased across the subsequent days (FR1: 2 days, FR5: 2 days; FR10: 2 days). All sessions were $30 \mathrm{~min}$ in duration and there was no limit to the number of reinforcers obtained during the sessions. After two days at FR10, a 30-min extinction session was instituted (no reinforcement was delivered for lever responding). Responses on both of the levers, total reinforcers earned, and responding in the magazine were programmed to be recorded.

\section{Conditioned Reinforcement Testing}

In the fourth experiment, another group of experimentally naive rats was first trained to associate a tone + light pairing with the delivery of reward (i.e., stimulusreward learning). During the first two sessions in the operant box (identical to the one described above), 20 water reinforcers were delivered randomly over a 20min session. In the next 10 sessions (20 min in duration), the subjects experienced the random presentation of 20 water reinforcers which were preceded by $5 \mathrm{sec}$ by a tone and activation of a light over the magazine. No levers were present in the chamber during training.

After the 10 training sessions, the subjects were repeatedly exposed to PCP or saline as detailed above. Following a one-week washout period, the subjects underwent two more 20-min sessions during which the tone + light stimulus signaled the presentation of the water reward. On the following day, responding on one novel lever, the active lever, was reinforced by the presentation of the tone + light stimulus alone (conditioned reinforcement or CR lever); no water was delivered during the test session. Responding on the novel inactive lever had no programmed consequences (no CR lever or NCR). Responses made on either lever, total reinforcers earned and the duration of head entries into 
the magazine were measured during the session. Test sessions were $30 \mathrm{~min}$ from the first CR lever response (see Taylor and Horger 1999).

\section{Statistical Methodology}

For the discrimination acquisition and reversal experiments, repeated measures analysis of variance were used to determine effects of group (drug treatment) across the multiple days of testing (the repeated measure). The dependent variable was the average number of errors per testing day. For the operant experiments, one-way ANOVA with Scheffe's F-test were used to determine group effects on the measures detailed above. All analyses were performed using Statview v4.02 (Abacus Concepts, Berkeley, CA) running on a Power Macintosh. The data are expressed as mean errors \pm standard error measure.

\section{RESULTS}

\section{Experiment 1: Acquisition of a Novel Discrimination}

In the first experiment, subjects were repeatedly exposed to saline or PCP for seven days, and following a oneweek withdrawal period, all subjects were trained to perform a novel visual discrimination. Both groups learned the visual discrimination within the five-day testing period (Figure 1). There was no significant effect of group $\left(\mathrm{F}_{(1,14)}=1.07, p=.32\right)$, nor was there a significant group $\mathrm{X}$ day interaction $\left(\mathrm{F}_{(4,56)}=0.31, p=.87\right)$. Thus, the drugtreated rats acquired the novel discrimination with the same degree of efficiency as the control subjects.

\section{Experiment 2: Discrimination Reversal Learning}

In Experiment 2, all subjects first learned a novel visual discrimination, and subsequently, the subjects were repeatedly exposed to either PCP or saline. Following withdrawal, the subjects were tested under reversal conditions (the reinforcement contingencies for the concurrent discrimination were reversed). There was a significant effect of drug treatment on performance across the 6 sessions of the reversal learning period (Figure 2 $\left(\mathrm{F}_{(1,14)}=93.84, p<.0001\right)$ and there was a group $\times$ day interaction $\left(\mathrm{F}_{(5,70)}=4.89, p=.001\right)$.

Although there were no differences between the groups on Day 1 of reversal learning (since both groups of subjects made errors), the PCP-treated subjects made more errors during reversal on Days 2-5. Both groups did reach a high level of performance, such that by the sixth session there were no significant differences between the groups. Thus, reversal learning was delayed, rather than prevented, after PCP treatment, as evidenced by the rightward shift in the learning curve in PCP-treated subjects.

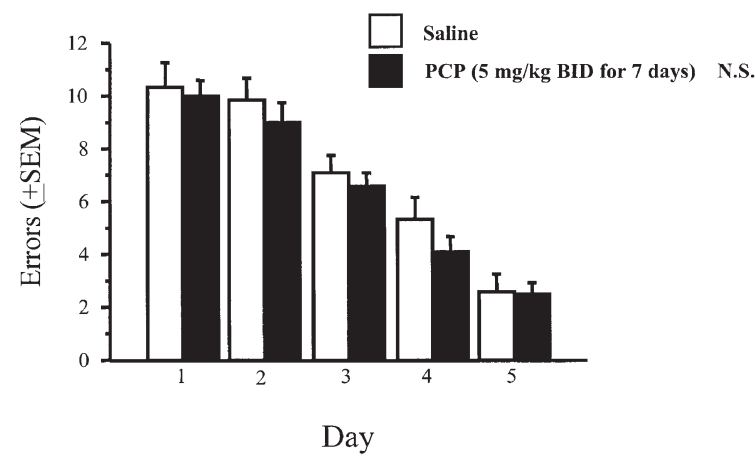

Figure 1. Acquisition of a Novel Discrimination. Acquisition of a simultaneous (black/white) discrimination is unaffected after subchronic PCP treatment. Repeated measures ANOVA (group being the factor and day being the repeated measure) showed no main effect of pre-treatment: $F(1,14)=$ $1.07, p=.32$, indicating that prior exposure to PCP failed to affect discrimination learning, i.e., acquisition of a stimulusreward association. N.S. indicates not significant by the PCP group. Means and S.E.M.'s of $n=8$ /group are shown.

\section{Experiment 3: Acquisition and Extinction of an Instrumental Response}

In the third experiment, all subjects were repeatedly exposed to PCP or saline, and following a one-week withdrawal period, operant testing was begun. On the first day, Pavlovian approach behavior was trained. There was a significant effect of drug treatment on the time spent in the magazine during as shown by a group $\times$ head entry interaction (Figure 3$)\left(\mathrm{F}_{(5,30)}=31.40, p<\right.$

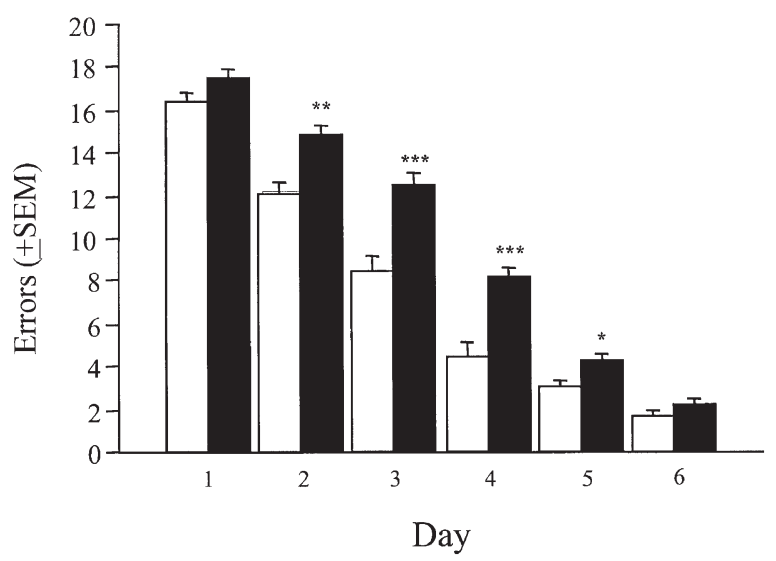

Figure 2. Discrimination Reversal Learning. Reversal of a simultaneous (black/white) discrimination is impaired after subchronic PCP treatment. Repeated measures ANOVA (group being the factor and day being the repeated measure) showed an interaction between pre-treatment group and day: $F(5,70)=4.58, p=.001$ indicating that in subjects that had already learned the stimulus-reward association, subsequent treatment with PCP impaired reversal learning. Post hoc analyses showed significant differences between the groups on Days $2-5\left(^{* * *} p<.005,{ }^{* *} p<.01{ }^{*} p<.05\right)$. Means and S.E.M.'s of $n=8$ /group are shown. 

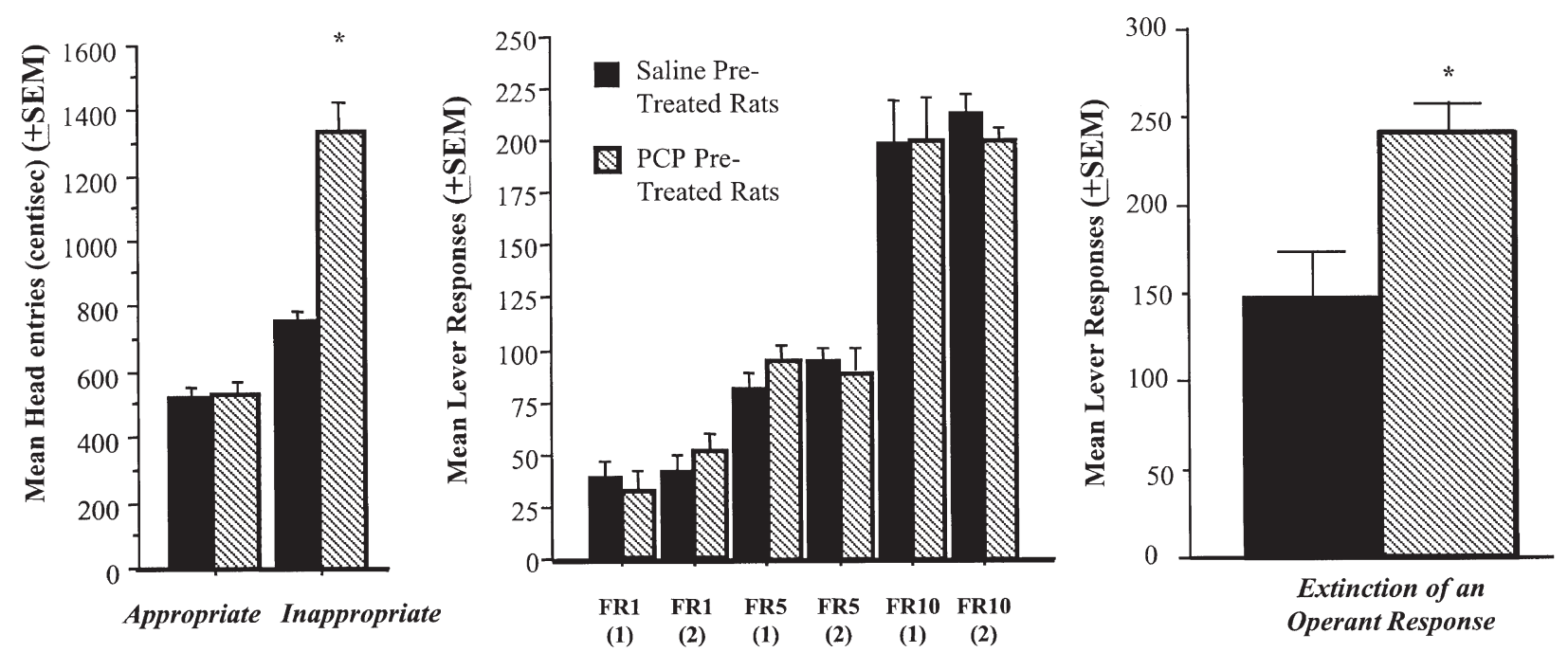

Figure 3. Fixed Ratio Responding and Extinction. Left: Animals treated subchronic PCP showed more inappropriate, but not appropriate, head entries into the magazine during the initial Pavlovian approach training $\operatorname{session}\left(\mathrm{F}(1,6)=31.40{ }^{*} p=\right.$ .002). Middle: No differences were found during fixed ration (FR) responding after subchronic PCP treatment. The value of the ratio and the session is shown below in parentheses. Right: Extinction of operant FR responding was delayed by previous exposure to PCP. PCP pre-treated rats responded significantly more on the lever after responding was no longer reinforced $\left(\mathrm{F}(1,6)=21.09 .{ }^{*} p=.005\right)$. Means and S.E.M.'s of $n=4$ /group are shown.

.002). Although PCP-treated subjects spent the same amount of time responding in the magazine during the "appropriate" times, as did the control subjects, they spent approximately twice as much time making "inappropriate" head entries during the training session.

On subsequent days, operant testing was begun. Repeated measures ANOVA for group effects at each stage (session) of fixed ratio performance (FR1, FR5, FR10) revealed no significant differences between PCPtreated and control groups or a group $\times$ day interaction, as both groups produced equivalent numbers of responses on the active lever when primary reward was delivered (Figure 3). Both groups of subjects showed increases in responding as the FR was increased across sessions $\left(\mathrm{F}_{(5,30)}=117.13, p<.001\right)$. Consequently, the numbers of reinforcers earned by the two groups were not significantly different. Moreover, there were no differences between the two groups in the number of responses delivered on the inactive lever.

When the schedule of reinforcement was changed to elicit extinction of bar pressing (neither primary reinforcement nor the tone + light stimulus was delivered), a significant effect of group was evident, as shown in Figure 3. That is, PCP-treated subjects produced more lever responses despite the absence of primary reinforcement than did control subjects $\left(\mathrm{F}_{(1,6)}=21.09, p<\right.$ .005). There were no group effects for responses on the inactive lever. Although this experiment was conducted with a small number of subjects $-n=4$ per group-the effect of PCP treatment on producing more responses during extinction were highly significant.

\section{Experiment 4: Responding for Conditioned Reinforcement}

In the fourth experiment, the subjects were first trained to associate the delivery of a primary reward (water) with a tone + light compound stimulus over 10 daily training sessions. Following this stimulus-reward training, the subjects were treated repeatedly with saline or PCP, and following a one week washout period, they were again subjected to two sessions of pairing of the compound stimulus with primary reward. No differences in approach to the magazine during either "appropriate" or "inappropriate" times were noted between groups when training preceded chronic treatment.

Following these two post-drug training sessions, responding on the novel "active" (CR) lever was associated contingently with the delivery of the compound stimulus alone (no water reinforcement was given). PCP-treated rats produced significantly more responses on the active CR lever than did control subjects (Figure 4) $\left(\mathrm{F}_{(1,10)}=10.70, p<.01\right)$. Reponses on the novel inactive lever (Figure 4; NCR) and head entries into the magazine were unaffected by drug treatment.

\section{DISCUSSION}

The current data support the notion that long-term exposure to PCP produces persistent impairments in the ability to modulate behavior based upon changing relationships between conditioned stimuli and reinforce- 


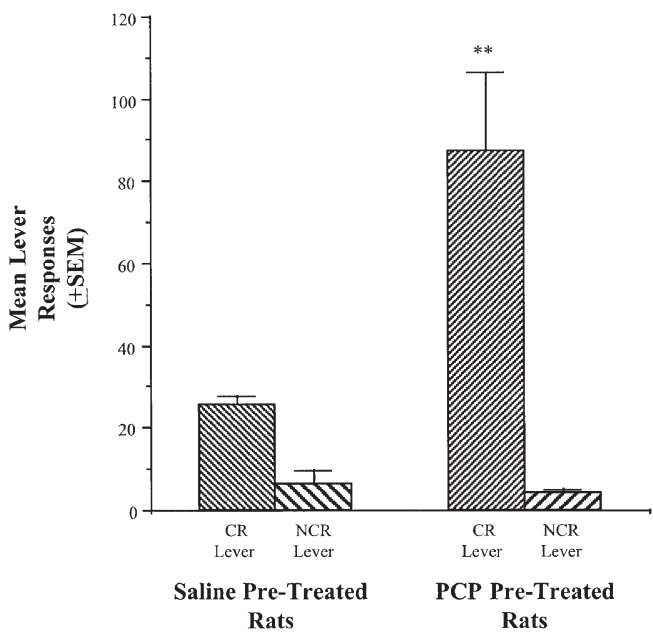

Figure 4. Responding for Conditioned Reinforcement. Subchronic PCP pretreatment produces enhanced responding for a conditioned reinforcer, $\mathrm{F}(1,10)=10.70{ }^{* *} p=.01$, compared with saline pre-treated rats, indicating that prior treatment with PCP enhances responding for conditioned reinforcement. Both groups of subjects showed increased responding on the CR lever compared with the NCR lever. Means and S.E.M.'s of $n=6$ /group are shown.

ment. PCP treatment did not affect the learning of a new association between response and reward (e.g., a novel concurrent discrimination or a novel instrumental response) but impaired the ability to shift responding away from a previously rewarded, but currently not rewarded, stimulus (e.g., discrimination reversal or extinction of an instrumental response). Moreover, the acquisition of a new response which is reinforced only by presentation of a conditioned, but not primary, reward is exaggerated in PCP-treated rats, indicating that these effects may be mediated, in part, by an increased motivational significance or control over behavior associated with conditioned reinforcing stimuli. Each of these behavioral deficits is indicative of deficits in inhibitory control and is consistent with behavioral impairments produced by damage to the frontal cortex.

Previously, we showed in rodents that this dose of PCP, schedule of its administration and period of withdrawal led to a selective reduction in basal and stressevoked dopamine-utilization in the prefrontal cortex and deficits in spatial working memory (see Jentsch et al. 1997b, 1998), effects that may be due, in part, to inhibitory control deficits (see Jentsch et al. 1997b). Subchronic exposure to PCP in monkeys also produces alterations in dopaminergic utilization in selected regions of prefrontal cortex and deficits in inhibitory control, as measured by impairments on the object retrieval/detour task (Jentsch et al. 1997a, 1999, 2000).

These PCP-induced behavioral and neurochemical deficits in rats and monkeys have been found up to 3-4 weeks after cessation of the subchronic treatment; however, whether these long-lasting alterations are permanent is not known. Nevertheless, these dopaminergic abnormalities produced by subchronic (intermittent) PCP treatment appear not to be attributable to neurotoxicity and resultant anatomical change and no evidence for PCP-induced alterations in dopamine levels have been reported (see Jentsch et al. 1998, for discussion). Nonetheless, PCP can at some high doses and/or after chronic treatment regimens produce such changes (Ellison 1995; Olney et al. 1989, 1991). Moreover, subchronic PCP-induced behavioral impairments can be ameliorated by several pharmacological manipulations hypothesized to alter dopaminergic neurotransmission (e.g., Jentsch et al. 1997a). Future studies will be aimed at addressing issues such the time course of these deficits and whether they are permanent and/or reversible.

\section{Relevance to Inhibitory Control Processes}

A variety of psychological impairments could be argued to be responsible for the behavioral deficits exhibited by PCP-treated rats and monkeys. We have hypothesized that impairments in inhibitory control may be characteristic of these behavioral abnormalities. It seems unlikely that other important psychological constructs required for accurate or optimum performance of these tasks, including instrumental learning, motor processes, selective attention, visual and spatial cognition or response planning, are impaired after PCP treatment because we observed no impairments in acquisition of a novel discrimination or acquisition of a novel instrumental response. Moreover, we did not find that PCP-treated rats showed abnormal responding on inactive (non-reinforced) levers during fixed ratio or conditioned reinforcement paradigms.

Impairments in task performance were found only when the animals were required to alter responding based upon a change in reinforcement contingencies, and responding in each of these paradigms was specifically directed towards previously rewarded stimuli. As such, it appears that PCP treatment produced deficits in the ability to inhibit previously conditioned, pre-potent responses. This is argued to be the basis of response inhibition, a psychological construct associated with the frontostriatal system (Dias et al. 1996a,b; 1997; Robbins 1996; Diamond 1988, 1996; Damasio 1996; Jentsch et al. 1997a, 2000; Jentsch and Taylor 1999; Taylor et al. $1990 a, b)$. Thus the impairments produced by PCP treatment appeared to be specific to a loss of inhibitory control when the "gating" or modulation of conditioned behavior is critical.

It is important to also consider the possible role of working memory in the observed task impairments. Goldman-Rakic (1996) has argued that working memory is the primary function of prefrontal cortex and that 
working memory for different sensory or functional domains is processed in parallel across the anatomical subdivisions of the frontal lobe. She has further hypothesized that impulsivity, perseveration and pre-potent responding can result from a failure of working memory. That is, in the absence of regulation of responding by working memory, the "default" is to exhibit conditioned, over-learned behavior. This hypothesis posits that inhibitory control is not an active process of prefrontal cortex, but rather, a result of deficits in working memory. Alternatively, long-term exposure to PCP or frontal lobe lesions produce deficits on an object retrieval/detour task (Diamond 1988; Jentsch et al. 1997a, 2000; Dias et al. 1996b), a task that likely requires response inhibition but only a modicum of working memory function since the "correct" response is always visible to the animal (Diamond 1988, 1996).

\section{Implications for Frontal Lobe Dysfunction}

The observed task-related impairments are reminiscent of those observed after lesions to the frontal cortex in both rodents and non-human primates. As noted above, ablative or excitotoxic lesions of the frontal cortex in rodents or macaques can produce impairments of discrimination learning and of extinction (Butter 1968; Iversen and Mishkin 1970; Divac 1971; Kolb et al. 1974; Rosenkilde 1975; Diamond 1988; Dias et al. 1996a,b, 1997; Wallis et al. 1997; Bussey et al. 1997). Moreover, frontal cortex lesions can produce exaggerated control of behavior by conditioned reinforcers and impair extinction (Weissenborn et al. 1997). Taken together with the current results, these data suggest that chronic exposure to PCP can produce impairments of a subset of the cognitive functions of the frontal cortex that are related specifically to inhibitory control.

\section{Implications for Motivated Behavior and Affective Dysfunction}

Repeated administration of PCP produces persistent dopaminergic dysfunction in the prefrontal cortex (Jentsch et al. 1997a, 1999), including the ventromedial, prelimbic frontal cortex (frontal areas 13/14/25/32/33) which densely innervates the nucleus accumbens (Haber et al. 1995). The nucleus accumbens and its dopaminergic innervation are known to be involved in reward processes (e.g., Everitt and Robbins 1992; Koob 1992; LeMoal and Simon 1991; Nestler 1994), and conditioned reinforcement, in particular (Taylor and Robbins 1984, 1986). Notably, reductions in cortical dopamine function can lead to increased nucleus accumbens dopamine transmission (Deutch et al. 1990; Deutch 1992). Recently, we found that chronic PCP treatment can produce heightened dopaminergic function in the nucleus accumbens in addition to cortical dopaminer- gic hypofunction in rodents (Jentsch et al. 1998) and frontal-cortical cognitive function in monkeys (Jentsch et al. 1997a, 2000).

We thus speculate that repeated exposure to drugs of abuse such as PCP produces dysfunction of corticolimbic circuits associated with behavioral inhibition and reward, which may result in "impulsivity" and exaggerated control of behavior by reward-related stimuli (Jentsch and Taylor 1999). This hypothesis of frontal cortical and mesolimbic dysfunction is further supported by the recent findings that repeated exposure to other drugs-of-abuse, i.e., amphetamine, cocaine, or heroin, can produce augmented responding for conditioned reinforcement (Cunningham et al. 1997; Taylor and Horger 1999) and deficits in frontal-cortical cognitive function, typified by a loss of inhibitory control (Rogers et al. 1999). Alterations in prefrontal cortical neurochemistry and function produced by drugs of abuse may thus lead to impaired cortical gating or modulation of reward-motivated behaviors that are dependent on the ventral striatum (Jentsch and Taylor 1999).

\section{CONCLUSIONS}

Here, we found that animals that received subchronic PCP treatment subsequently display impulsivity when tested on tasks that require inhibiting a conditioned response when stimulus-reward contingencies have changed. Based on our previous studies, we suggest that the deficits observed in the present studies were due to PCP-induced dysfunction within the corticostriatal system. Likewise, animals with putative PCP-induced activation of the ventral striatum showed enhanced control of behavior by stimuli associated with reward. Because subcortical function is modulated by the frontal cortex, we hypothesize that deficits in cortical response inhibition mechanisms may produce dysregulation of subcortical-reward mechanisms, leading to repetition of previously-rewarded responses.

Several psychiatric diseases, most obviously drug abuse, schizophrenia, and obsessive-compulsive disorders, are characterized by increased impulsivity. Each of these pathological states is associated with profound inability to inhibit inappropriate or maladaptive tendencies or behaviors. Deficits in response inhibition can be observed both at the cognitive level, where mental flexibility and attentional shifting are required, and at basic reward level, where stimuli with incentive value compete for the control of behavior. The neural dysfunction subserving impulsivity in psychiatric patients is not understood, but it is an issue of critical importance because pharmacological therapies that target impulsivity are largely ineffective. Further studies examining monoaminergic influences on response 
inhibition processes are necessary to elucidate the neurochemical basis of the observed behavioral impairments and to determine whether pharmacological treatments can reverse or ameliorate these behavioral deficits.

\section{ACKNOWLEDGMENTS}

Thanks to Kristina Kudelko, Ericka Rosner, and Valyphone Phantharangsy. Robert H. Roth is gratefully acknowledged for his continued support. Supported, in part, by USPHS grants DA11717 (JRT) and USPHS MH57483 (RHR).

\section{REFERENCES}

Bussey TJ, Muir JL, Everitt BJ, Robbins TW (1997): Dissociable effects of anterior and posterior cingulate lesions on the acquisition of a conditional discrimination: Facilitation of early learning vs. impairment of late learning. Behav Brain Res 82:31-44

Butter CM (1968): Perseveration in extinction and in discrimination reversal following selective frontal ablations in Macaca mulatta. Physiol Behav 4:163-171

Cunningham ST, Finn M, Kelley AE (1997): Sensitization of the locomotor response to psychostimulants after repeated opiate exposure: Role of the nucleus accumbens. Neuropsychopharmacology 16:147-155

Damasio AR (1996): The somatic marker hypothesis and the possible functions of the prefrontal cortex. Phil Trans R Soc Lond B 351:1413-1420

Deutch AY (1992): The regulation of subcortical dopamine systems by the prefrontal cortex: Interactions of central dopamine systems and the pathogenesis of schizophrenia. J Neural Trans 36(Suppl):61-89

Deutch AY, Clark WA, Roth RH (1990): Prefrontal cortical dopamine depletion enhances the responsiveness of mesolimbic dopamine neurons to stress. Brain Res 521:311-315

Diamond A (1988): Differences between adult and infant cognition: Is the crucial variable presence or absence of language? In Weiskrantz L (ed), Thought without Language. Oxford, Clarendon Press, pp 337-370

Diamond A (1996): Evidence for the importance of dopamine for prefrontal cortex functions early in life. Phil Trans R Soc Lond B 351:1483-1494

Dias R, Robbins TW, Roberts AC (1996a): Dissociation in prefrontal cortex of affective and attentional shifts. Nature 380:69-72

Dias R, Robbins TW, Roberts AC (1996b): Primate analogue of the Wisconsin card sorting test: Effects of excitotoxic lesions of the prefrontal cortex in the marmoset. Behav Neurosci 110:872-886

Dias R, Robbins TW, Roberts AC (1997): Dissociable forms of inhibitory control within prefrontal cortex with an analog of the Wisconsin Card Sort test-restriction to novel situations and independence from on-line processing. J Neurosci 17:9285-9297
Divac I (1971): Frontal lobe system and spatial reversal in the rat. Neuropsychologia 9:175-183

Ellison (1995): The N-methyl-D-aspartate antagonists phencyclidine, ketamine, and dizocilpine as both behavioral and anatomical models of the dementias. Brain Res Rev 20:250-267

Everitt BJ, Robbins TW (1992): Amygdala-ventral striatal interactions in reward-related processes. In Aggelton JP (ed), The Amygdala. New York, NY, Wiley-Liss Inc., pp 401-409

Goldman-Rakic PS (1996): The prefrontal landscape: Implications of functional architecture for understanding human mentation and the central executive. Phil Trans R Soc Lond B 351:1445-1453

Haber SN, Kunishio K, Mizobuchi M, Lynd-Balta E (1995): The orbital and medial prefrontal circuit through the primate basal ganglia. J Neurosci 15:4851-4867

Hill RT (1970): Facilitation of conditioned reinforcement as a mechanism of psychomotor stimulation. In Costa E, Garattini S (eds), Amphetamines and Related Compounds. New York, Raven Press, pp 781-795

Iversen SD, Mishkin M (1970): Perseverative interference in monkeys following selective lesions of the inferior prefrontal convexity. Exp Brain Res 11:376-386

Jentsch JD, Redmond DE Jr, Elsworth JD, Taylor JR, Youngren KD, Roth RH (1997a): Enduring cognitive dysfunction and cortical dopamine deficits in monkeys after long-term administration of phencyclidine. Science 277:953-955

Jentsch JD, Tran A, Le D, Youngren KD, Roth RH (1997b): Subchronic phencyclidine administration reduces mesoprefrontal dopamine utilization and impairs prefrontal cortical-dependent cognition in the rat. Neuropsychopharmacology 17:92-99

Jentsch JD, Taylor JR, Roth RH (1998): Subchronic phencyclidine administration increases mesolimbic dopamine system responsivity and augments stress- and amphetamine-induced hyperlocomotion. Neuropsychopharmacology 19:105-113

Jentsch JD, Taylor JR, Elsworth JD, Redmond DE Jr, Roth RH (1999): Altered frontal cortical dopaminergic transmission in monkeys after subchronic phencyclidine exposure: Involvement of frontostriatal cognitive deficits. Neuroscience 90:823-832

Jentsch JD, Taylor JR (1999): Impulsivity resulting from frontostriatal dysfunction in drug abuse: Implications for the control over behavior by reward-related stimuli. Psychopharmacology 146:373-390

Jentsch JD, Roth RH, Taylor JR (2000): Object retrieval/ detour deficits in monkeys produced by prior subchronic phencyclidine administration: Evidence for cognitive impulsivity. Biol Psychiatry 48:415-424

Kolb B, Nonneman AJ, Singh RK (1974): Double dissociation of spatial impairments and perseveration following selective prefrontal lesions in rats. J Comp Physiol Psychol 87:772-780

Koob GF (1992): Neuronal mechanisms of drug reinforcement. Ann N Y Acad Sci 654:171-191

LeMoal M, Simon H (1991): Mesocorticolimbic dopaminergic network: functional and regulatory roles. Physiol Rev 71:155-234 
Mackintosh NJ (1974): The psychology of Animal Learning. London, Academic Press

Milner B (1963): Some effects of frontal lobe lesions in man. In Warren JM, Akert K (eds), The Frontal Granular Cortex and Behavior. New York, McGraw-Hill, pp 313-331

Mishkin M (1964): Perseveration of central sets after frontal lesions in monkeys. In Warren JM, Akert K (eds), The Frontal Granular Cortex and Behavior. New York, McGraw-Hill, pp 219-241

Murphy BL, Arnsten AFT, Goldman-Rakic PS, Roth RH (1996): Increased dopamine turnover in the prefrontal cortex impairs spatial working memory performance in rats and monkeys. Proc Natl Acad Sci U S A 93:13251329

Nestler EJ (1994): Molecular neurobiology of drug addiction. Neuropsychopharmacology 11:77-87

Olney JW, Labruyere J, Price MT (1989): Pathological changes in cerebrocortical neurons by phencyclidine and related drugs. Science 244:1360-1362

Olney JW, Labruyere J, Wang G, Wozniak DF, Price MT, Sesma MA (1991): NMDA receptor antagonist neurotoxicity: Mechanism and prevention. Science 254:15151518

Robbins TW (1975): The potentiation of conditioned reinforcement by psychomotor stimulant drugs: A test of Hill's hypothesis. Psychopharmacology (Berl) 45:103114

Robbins TW (1976): Relationship between reward-enhancing and stereotypical effects of psychomotor stimulant drugs. Nature 264:57-59

Robbins TW (1996): Dissociating executive functions of the prefrontal cortex. Phil Trans R Soc Lond B 351:14631471

Rogers RD, Everitt BJ, Baldacchino A, Blackshaw AJ, Swainson R, Wynne K, Backer NB, Hunter J, Carthy T, Booker E, London M, Deakin JWF, Sahakian BJ, Robbins TW (1999): Dissociable deficits in the decision-making cognition of chronic amphetamine abusers, opiate abusers, patients with focal damage to the prefrontal cortex, and tryptophan-depleted normal volunteers: Evidence for monoaminergic mechanisms. Neuropsychopharmacology 20:322-339

Rosenkilde CE (1975): Functional heterogeneity of the prefrontal cortex in the monkey: A review. Behav Neural Biol 25:301-345

Taylor JR, Robbins TW (1984): Enhanced behavioral control by conditioned reinforcers following microinjections of $d$-amphetamine into the nucleus accumbens. Psychopharmacology 84:564-576

Taylor JR, Robbins TW (1986): 6-hydroxydopamine lesions of the nucleus accumbens, but not the caudate nucleus, attenuate enhanced responding with reward-related stimuli produced by intra-accumbens $d$-amphetamine. Psychopharmacology 90:390-397

Taylor JR, Horger BA (1999): Enhanced responding for conditioned reward produced by intra-accumbens amphetamine is potentiated after cocaine sensitization. Psychopharmacology 142:31-40

Taylor JR, Elsworth JD, Roth RH, Sladek JR, Redmond DE Jr (1990a): Cognitive and motor deficits in the acquisition of an object retrieval/detour task in MPTP-treated monkeys. Brain 113:617-637

Taylor JR, Roth RH, Sladek JR, Redmond DE Jr (1990b): Cognitive and motor deficits in the performance of an object retrieval task with a barrier-detour in monkeys (Cercopithecus aethiops sabaeus) treated with MPTP: Long-term performance and effect of transparency of the barrier. Behav Neurosci 104:564-576

Wallis JD, Collins P, Everitt BJ, Robbins TW, Roberts AC (1997): Selective lesions of the prefrontal cortex in the marmoset produce dissociable effects on the object retrieval task. Soc Neurosci Abstr 23:1605

Weissenborn R, Robbins TW, Everitt BJ (1997): Effects of medial prefrontal or anterior cingulate cortex lesions on responding for cocaine under fixed-ratio and secondorder schedules of reinforcement in rats. Psychopharmacology 134:242-257 